\title{
Antibody-conjugated gold-gold sulfide nanoparticles as multifunctional agents for imaging and therapy of breast cancer
}

This article was published in the following Dove Press journal:

International Journal of Nanomedicine

22 June 2010

Number of times this article has been viewed

\section{Emily S Day \\ Lissett R Bickford \\ John H Slater \\ Nicholas S Riggall \\ Rebekah A Drezek \\ Jennifer L West}

Department of Bioengineering, Rice University, Houston, TX, USA
Correspondence: Jennifer LWest 6100 Main Street, MS-142,

Houston, TX 77005, USA

$\mathrm{Tel}+\mathrm{I} 7133485955$

Fax + I 7133485877

Email jwest@rice.edu

\begin{abstract}
The goal of this study was to develop near-infrared (NIR) resonant gold-gold sulfide nanoparticles (GGS-NPs) as dual contrast and therapeutic agents for cancer management via multiphoton microscopy followed by higher intensity photoablation. We demonstrate that GGS-NPs exposed to a pulsed, NIR laser exhibit two-photon induced photoluminescence that can be utilized to visualize cancerous cells in vitro. When conjugated with anti-HER2 antibodies, these nanoparticles specifically bind SK-BR-3 breast carcinoma cells that overexpress the HER2 receptor, enabling the cells to be imaged via multiphoton microscopy with an incident laser power of $1 \mathrm{~mW}$. Higher excitation power $(50 \mathrm{~mW})$ could be employed to induce thermal damage to the cancerous cells, producing extensive membrane blebbing within seconds leading to cell death. GGS-NPs are ideal multifunctional agents for cancer management because they offer the ability to pinpoint precise treatment sites and perform subsequent thermal ablation in a single setting.
\end{abstract}

Keywords: cancer, nanomedicine, multiphoton microscopy, photoluminescence, photothermal therapy, theranostics

\section{Introduction}

Development of superior diagnostic and therapeutic tools for cancer is crucial, as the age-adjusted mortality has remained relatively unchanged for the past 35 years despite use of currently available technologies. ${ }^{1}$ Researchers have recently begun to engineer multifunctional nanoparticles with properties suitable for both imaging and treatment of cancer in an effort to better manage the disease. ${ }^{2}$ Here we present GGS-NPs as a dual contrast and therapeutic agent when combined with two-photon microscopy. These nanoparticles strongly absorb NIR wavelengths of light which penetrate deeply into tissue, ${ }^{3}$ rendering them useful for NIR photothermal cancer therapy and optical imaging. Several gold-based NIR-absorbing nanoparticles, including silica-gold nanoshells, ${ }^{4-8}$ gold nanorods, ${ }^{9-12}$ and gold nanocages, ${ }^{13}$ have demonstrated the ability to convert incident light energy into heat sufficient to irreversibly damage targeted cancerous cells. A promising alternative to conventional treatment modalities, nanoparticle-assisted photothermal therapy is minimally invasive, highly effective, and anticipated to have limited side effects. Additionally, the ability to image nanoparticles prior to treatment will prevent inadvertent delivery of heat to healthy tissue by helping distinguish normal from diseased regions. Although optical coherence tomography ${ }^{4}$ and photoacoustic tomography ${ }^{14-16}$ have been evaluated with gold-based NIR-absorbing nanoparticles as contrast agents, these techniques are limited to wide field-of-view applications. A more 
promising imaging modality is multiphoton microscopy, which offers the advantage of providing high resolution at the microscopic level with the ability to obtain both functional and morphological information.

Nonlinear optics has historically been used to evaluate fluorescent signals in cell and animal model studies. ${ }^{17}$ Multiphoton microscopy incorporates an ultrafast pulsed laser to deliver two NIR photons simultaneously to fluorophores, thereby producing images with superior resolution since NIR light interferes minimally with tissue and only molecules at the focal plane of the laser become excited. Although fluorophores are the traditional contrast agents for two-photon microscopy, recent work has demonstrated that nanoparticles with strong surface plasmon resonance in the NIR can be employed as well. Advances in this research area were made following the discovery that roughened metal surfaces exhibit twophoton induced photoluminescence (TPL), a phenomenon attributed to coupling of light with localized plasmon resonances. ${ }^{18}$ Similarly, NIR-resonant nanoparticles excited with a pulsed laser luminesce when electrons in the sp-band recombine with holes in the d-band created following sequential absorption of single photons. ${ }^{19} \mathrm{TPL}$ of gold nanoshells and nanorods has been extensively studied and these nanoparticles display enhanced properties for multiphoton microscopy compared to traditional fluorophores, including brighter signals and increased resistance to photobleaching. ${ }^{10,12,20-25}$ Specifically, it has been demonstrated that the TPL signal of a single nanorod is nearly 60 times brighter than a single rhodamine $6 \mathrm{G}$ molecule, ${ }^{22}$ and nanoshells are approximately 140 times brighter than fluorescent beads and display brightness on the same order of magnitude as nanorods. ${ }^{21}$ The enhanced luminescent signal reported for gold-based nanoparticles compared to traditional fluorophores and the ease of conjugation of biomolecules to gold surfaces renders these materials ideal contrast agents for multiphoton microscopy.

In this work we combined imaging and therapy of targeted cancer cells in one system through implementation of two-photon microscopy in conjunction with functionalized, NIR-absorbing GGS-NPs. These nanoparticles were first developed by Zhou et al and described to have a core/shell structure. ${ }^{26}$ Zhang and colleagues then proposed a gold nanoparticle aggregate structure. ${ }^{27}$ Due to consequent debate about their composition, ${ }^{28-30}$ specific structural details must be further elucidated. Despite this, potential applications of these nanoparticles reported in the literature continue to grow. GGS-NPs have been used as drug carriers with minimal toxicity $^{31,32}$ and have more recently been described as a photothermal cancer therapeutic agent. ${ }^{33}$ Gobin et al found that GGS-NPs are as effective as silica-gold nanoshells at eliminating tumors in vivo by photothermal ablation while also possessing a better distribution profile, with a higher ratio of nanoparticle accumulation in tumors versus organs associated with the reticuloendothelial system. ${ }^{33}$ This finding can likely be attributed to the smaller size of GGS-NPs $(25-50 \mathrm{~nm}$ diameter) compared to silica-gold nanoshells (120-150 nm diameter). Together, the small size and apparent non-toxicity of GGS-NPs make them suitable as dual contrast and therapeutic agents for in vivo cancer management applications.

Here we demonstrate a proof-of-concept that GGS-NPs can be used simultaneously as targeted contrast agents and mediators of photothermal therapy by exploiting their optical properties and gold surface chemistry. GGS-NPs were specifically targeted towards SK-BR-3 breast carcinoma cells, which over-express the HER2 receptor, ${ }^{34}$ by functionalizing the gold surface with anti-HER2 antibodies. This is, to our knowledge, the first report that describes antibody conjugation to GGS-NPs and subsequent nanoparticle binding to targeted cancerous cells. Upon exposure to a pulsed, NIR laser these GGS-NPs demonstrated TPL and could therefore be used to visualize SK-BR-3 cells in vitro via multiphoton microscopy with an incident power of $1 \mathrm{~mW}$. In addition, increasing the power output of the excitation laser to $50 \mathrm{~mW}$ induced thermal damage to targeted cells and no damage to non-targeted cells. The ability to image these nanoparticles during their concurrent use as photothermal agents renders them highly attractive for use in cancer management, particularly in applications that require very specific therapy in order to maintain integrity of nearby vital regions of healthy tissue.

\section{Material and methods GGS-NP synthesis and functionalization}

GGS-NPs were synthesized using a variation of the procedures described by Averitt et a ${ }^{35}$ and Schwartzberg et al. ${ }^{30}$ Solutions of $\mathrm{HAuCl}_{4}\left(2 \mathrm{mM}\right.$, Alfa Aesar, Ward Hill, MA) and $\mathrm{Na}_{2} \mathrm{~S}_{2} \mathrm{O}_{3}$ ( $1 \mathrm{mM}$, Sigma, Saint Louis, MO) were prepared in milli-Q water, aged two days at room temperature, and mixed in small quantities at volumetric ratios ranging from 1:1 to 1:2 $\left(\mathrm{HAuCl}_{4}: \mathrm{Na}_{2} \mathrm{~S}_{2} \mathrm{O}_{3}\right)$. The ratio that produced nanoparticles resonant near $800 \mathrm{~nm}$ as determined with a UV-visible spectrophotometer (Cary 50, Varian, Walnut Creek, CA) was used to synthesize a large batch of nanoparticles for in vitro experiments. 
GGS-NPs were visualized with transmission electron microscopy and the diameter of at least 50 nanoparticles per sample was measured with ImageJ software $(\mathrm{NIH}$, Bethesda, MD). Calculation of the mean diameter revealed a slight batch-to-batch variability in average particle size, which ranged from $26 \mathrm{~nm}$ to $37 \mathrm{~nm}$. In the initial reaction solutions we also observed $\sim 5 \mathrm{~nm}$ diameter colloidal gold and 50-100 $\mathrm{nm}$ flat triangular nanoparticles; however, most of these particles were removed from solution by a multi-step centrifugation process so the final product used in experiments consisted of a majority of GGS-NPs. Dynamic light scattering was also incorporated to assess nanoparticle size and analysis of multiple batches with a ZetaSizer NanoZS (Malvern Instruments, Worcestershire, UK) revealed an average hydrodynamic diameter of $42.2 \mathrm{~nm}$, in good agreement with the transmission electron microscopy (TEM) results.

GGS-NPs were conjugated to either anti-HER2 (NeoMarkers, Freemont, CA) or nonspecific anti-IgG (Sigma) antibodies using 2,000 Da orthopyridyl-disulfide-poly(ethylene glycol)-N-hydroxysuccinimide (OPSS-PEG-NHS, Creative PEGWorks, Winston Salem, NC) as a linker. PEG-antibody conjugates were prepared by reacting one part $125 \mu \mathrm{M}$ OPSSPEG-NHS with 9 parts $1 \mathrm{mg} / \mathrm{mL}$ antibody at $4^{\circ} \mathrm{C}$ overnight. This reaction produces a stable amide bond between primary amines on the antibody and carboxyl groups on the PEG chain that are exposed when the NHS terminus is cleaved in water. The particles were suspended in milli-Q water and exposed to PEG-antibody conjugates for 1 hour at $4^{\circ} \mathrm{C}$ at a 100:1 volumetric ratio. Following antibody coupling, GGSNPs were reacted with a solution of mPEG-SH ( 5 mM, 5,000 Da, Laysan Bio, Inc., Arab, AL) for a minimum of 4 hours at $4{ }^{\circ} \mathrm{C}(1: 200$ volumetric ratio) to passivate any exposed gold surface area. GGS-NPs coated with mPEG-SH only (no antibody) were also synthesized for use as a negative control. Self-assembly of PEG-antibody and mPEG-SH onto the nanoparticle surface is possible due to dative interactions between sulfur and gold. Following antibody and/or PEG modification, GGS-NPs were centrifuged to remove unbound molecules, aspirated, and suspended in phosphate buffered saline (PBS) at an optical density of $2.0\left(\sim 4.2 \times 10^{11}\right.$ particles/ $\mathrm{ml})$ unless otherwise noted.

\section{Quantification of antibody bound to nanoparticles}

To verify that antibody and mPEG-SH successfully bound the GGS-NP surface we monitored changes in hydrodynamic diameter, which should increase upon addition of biomolecules. Using a Malvern ZetaSizer NanoZS, four nanoparticle formulations were studied: (1) Bare GGS-NPs, (2) GGS-NPs coated with mPEG-SH, (3) GGS-NPs coated with mPEG-SH and anti-IgG antibodies, and (4) GGS-NPs coated with mPEG-SH and anti-HER2 antibodies. The mean hydrodynamic diameter of each formulation was calculated as an average from three sets of nanoparticles, with individual sets also being tested in triplicate. Bare nanoparticles demonstrated a mean hydrodynamic diameter of $42.2 \mathrm{~nm}$, which increased to $58.0 \mathrm{~nm}$ upon addition of mPEG-SH. A further increase occurred when either antibody formulation was included, with diameter of $69.8 \mathrm{~nm}$ for anti-IgG coated nanoparticles and $63.4 \mathrm{~nm}$ for anti-HER2 coated nanoparticles. These results suggested the antibody and $\mathrm{mPEG}-\mathrm{SH}$ were able to self-assemble on the nanoparticle surface using the disulfide or thiol terminus, respectively.

To quantify the amount of antibody present on the nanoparticles, targeted (coated with $\mathrm{mPEG}-\mathrm{SH}$ and antibody) and control (only mPEG-SH coated) nanoparticles were incubated with $10 \mu \mathrm{g} / \mathrm{ml}$ horseradish peroxidase (HRP)conjugated goat anti-mouse IgG (for quantification of mouse anti-human HER2) or HRP-conjugated rabbit anti-goat IgG (for quantification of goat anti-mouse IgG) (both HRP antibodies from Sigma). Nonspecific reaction sites were blocked with a $3 \%$ solution of bovine serum albumin (BSA, Sigma) in PBS. To remove unbound HRP-labeled antibodies, the nanoparticles were centrifuged twice at $1500 \mathrm{~g}$ for eight minutes and suspended in 3\% BSA. The HRP bound to GGS-NPs was developed with 3,3',5,5'-tetramethylbenzidine dihydrochloride (Sigma) for 15 minutes and the reaction was stopped by addition of $2 \mathrm{M}$ sulfuric acid. The developed HRP was compared to a standard curve of the appropriate HRP-conjugated anti-IgG by determining the absorbance at $450 \mathrm{~nm}$ with a spectrophotometer. The total amount of peroxidase-labeled anti-IgG present in solution was divided by the total number of GGS-NPs in solution to determine the number of antibodies per nanoparticle. The number of nanoparticles was calculated from the Beer-Lambert law with the extinction coefficient of GGS-NPs derived from Mie theory as described by Averitt et al. ${ }^{35}$

\section{Cell culture}

SK-BR-3 breast carcinoma cells (American Type Culture Collection, Manassas, VA), which over-express the HER2 receptor, ${ }^{34}$ were cultured in McCoy's $5 \mathrm{~A}$ growth medium (Sigma) containing 10\% fetal bovine serum and 1\% penicillin-streptomycin at $37^{\circ} \mathrm{C}$ in a $5 \% \mathrm{CO}_{2}$ environment. For experiments, cells transferred to $15 \mathrm{~mL}$ conical tubes 
$\left(6 \times 10^{5}\right.$ cells/tube) were centrifuged (115 g, 3 minutes $)$ to form pellets that were subsequently suspended in $1 \mathrm{~mL}$ of one of the four following solutions: (1) nanoparticles coated with anti-HER2 and mPEG-SH, (2) nanoparticles coated with anti-IgG and mPEG-SH, (3) nanoparticles coated with only mPEG-SH, or (4) PBS. The nanoparticle solutions consisted of approximately $4.2 \times 10^{11}$ GGS-NPs. Cells were incubated in these suspensions for 30 minutes at $37^{\circ} \mathrm{C}$ in a hybridization chamber (VWR International, West Chester, PA) with constant rotation at $7 \mathrm{rpm}$. Following incubation, the samples were centrifuged (115 g, 3 minutes), aspirated, and diluted in PBS to remove any particles not bound to the cells. This rinsing procedure was repeated thrice followed by resuspension in growth media $(1 \mathrm{~mL})$. The cells were cultured on chambered coverglass overnight before experiments were performed. For studies of the effect of thermal therapy on cell membrane structure, the cells were labeled with $5 \mu \mathrm{M}$ DiI (Molecular Probes, Eugene, OR) for 10 minutes prior to incubation with the nanoparticles as described above.

\section{Multiphoton microscopy and photothermal therapy}

A Zeiss Laser Scanning Microscope (LSM) 510 META (Carl Zeiss, Inc., Thornwood, NJ) equipped with a femtosecondpulsed Ti:sapphire laser source (Chameleon, Coherent, Inc., Santa Clara, CA) was used to perform multiphoton microscopy and photothermal therapy experiments. The wavelength of the output laser beam was tuned to match the peak extinction of the GGS-NPs and operated with a pulse width of $140 \mathrm{fs}$ and repetition rate of $90 \mathrm{MHz}$. A short-pass dichroic mirror was used to reflect incident NIR light onto the sample through a $20 \times$ objective (numerical aperture $(N A)=0.75$ ) or a $63 \times$ objective $(\mathrm{NA}=1.4)$ and to collect photoluminescence.
Background signal was reduced with an infrared-blocking filter and the META detector was used to collect TPL from the GGS-NPs between 451-644 nm.

For imaging nanoparticles on cells, incident laser power was $1 \mathrm{~mW}$ with a pixel dwell time of $12.8 \mu \mathrm{sec}$ and the laser beam was raster-scanned across a $450 \mu \mathrm{m} \times 450 \mu \mathrm{m}$ area. Calculating laser intensity by dividing power by the area of the Airy disc, this corresponds to a fluence of $0.96 \mathrm{~J} / \mathrm{cm}^{2}$. To perform photothermal ablation, the samples were repositioned and laser power was increased to $50 \mathrm{~mW}\left(48.1 \mathrm{~J} / \mathrm{cm}^{2}\right)$ while maintaining the same dwell time. Samples were treated with a single pass of the laser. One hour after laser treatment cell viability was assessed by labeling cells with Calcein AM (1 $\mu \mathrm{M}$, Molecular Probes, Eugene, OR), a live cell stain, and ethidium homodimer-1 ( $4 \mu \mathrm{M}$, EthD-1, Molecular Probes), a dead cell stain. Fluorescence microscopy was performed with an inverted Zeiss Axiovert 135 phase contrast microscope (Carl Zeiss).

\section{Results \\ GGS-NP characterization}

Visualization of GGS-NPs with transmission electron microscopy (Figure 1a) and subsequent analysis with ImageJ software revealed slight batch-to-batch variability in nanoparticle size, with the smallest batch of nanoparticles produced having a diameter of $26 \pm 2 \mathrm{~nm}$ and the largest having diameter of $37 \pm 4 \mathrm{~nm}$. Variations in nanoparticle diameter did not affect microscopy or photoablation results when experiments were repeated using separate nanoparticle batches. Dynamic light scattering revealed an average hydrodynamic diameter of $42.2 \mathrm{~nm}$, in good agreement with the TEM results. GGS-NPs are thus less than one-third the size of silica-gold nanoshells, ${ }^{36}$ and similar in size to nanorods ${ }^{37,38}$
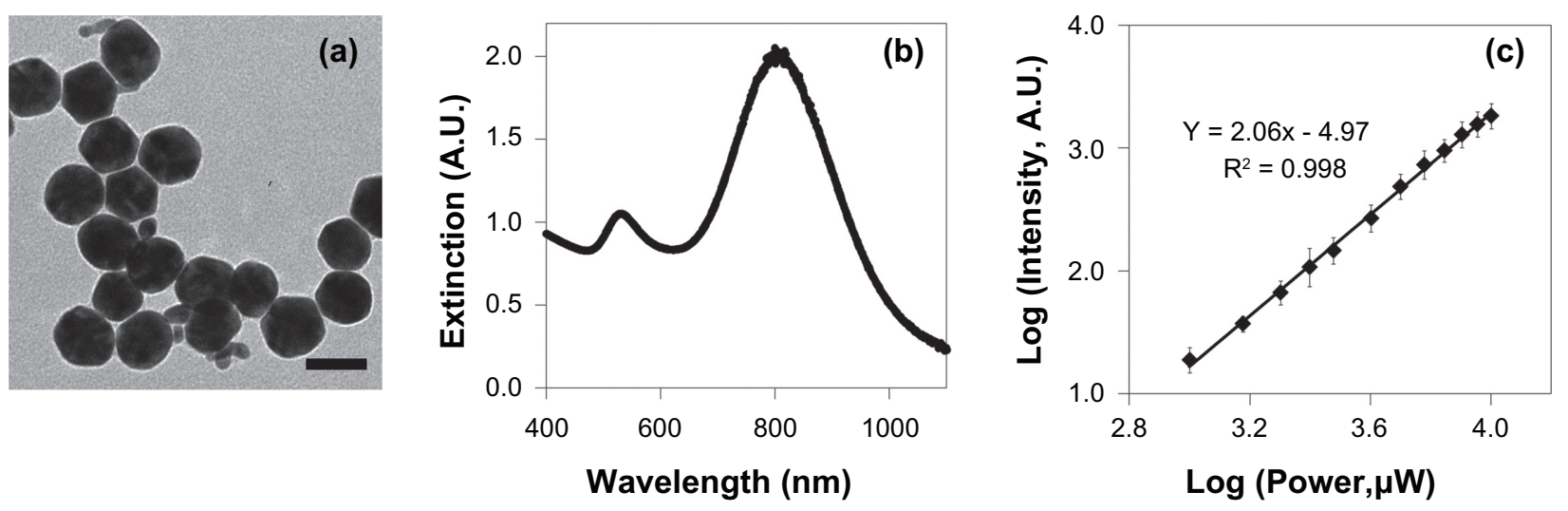

Figure I a) TEM of GGS-NPs. Scale bar $=40 \mathrm{~nm}$. b) Extinction spectrum of the GGS-NPs. c) GGS-NPs displayed a quadratic dependence of luminescence intensity on excitation power when exposed to an $800 \mathrm{~nm}$ pulsed laser. 
and nanocages. ${ }^{39}$ Although $\sim 5 \mathrm{~nm}$ diameter colloidal gold and 50-100 nm flat triangular nanoparticles were present in the initial nanoparticle solution, most of these particles were removed by centrifugation so the final product used in experiments consisted of a majority of GGS-NPs. Extinction characteristics were determined with a spectrophotometer and GGS-NPs had a peak plasmon resonance centered around $800 \mathrm{~nm}$ with a second extinction peak present at $530 \mathrm{~nm}$ due to remaining colloidal gold (Figure $1 b$ ). These findings are consistent with the synthesis and purification of GGS-NPs described by Gobin et al. ${ }^{33}$

Antibody coverage was quantified with a modified enzyme-linked immunosorbent assay (ELISA) and both anti-HER2 and anti-IgG coated GGS-NPs bound significantly more HRP-antibodies than PEG-coated GGS-NPs ( $P<0.01$ for both). The ELISA on anti-HER2 GGS-NPs indicated 55.0 \pm 4.0 antibodies per nanoparticle while control GGS-NPs remained free of antibody $(-1.3 \pm 2.6$ antibodies/ nanoparticle). Similar antibody densities were obtained for anti-IgG GGS-NPs compared to control GGS-NPs, with targeted nanoparticles bearing $32.6 \pm 3.2$ antibodies per nanoparticle and control GGS-NPs showing negligible background ( $-1.1 \pm 1.5$ antibodies/nanoparticle).

\section{GGS-NPs exhibit two-photon induced photoluminescence}

The ability of GGS-NPs to produce TPL was probed by imaging particles in aqueous solution with the multiphoton microscope with excitation power ranged from 1-10 $\mathrm{mW}$. The average emission intensity was determined using Image J software (NIH) and plotted versus excitation power. A quadratic dependence of nanoparticle emission intensity as a function of incident laser power was observed, with the slope of the fit linear curve being $2.06 \pm 0.03$, indicating a two-photon absorption process (Figure 1c). ${ }^{20-23}$ To compare brightness of the GGS-NP signal with reported values for other nanoparticle formulations we also synthesized and imaged spherical nanoshells consisting of a $120 \mathrm{~nm}$ diameter silica core and a $14.5 \mathrm{~nm}$ thick gold shell (mean particle diameter $=149 \mathrm{~nm}$ ) using the procedure introduced by Oldenburg et al. ${ }^{36}$ Silica-gold nanoshells and GGS-NPs were prepared in aqueous solution to optical density (OD) 10 at $800 \mathrm{~nm}$ and $500 \mu \mathrm{L}$ of each nanoparticle solution was placed in chambered coverglass. The samples were imaged with $1 \mathrm{~mW}$ and $10 \mathrm{~mW}$ excitation power at $800 \mathrm{~nm}$ and the mean intensity per particle was determined using Image J software $(\mathrm{NIH})$. Analysis of several hundred particles of each type revealed that at $1 \mathrm{~mW}$ excitation, the brightness ratio of GGS-NPs to nanoshells was $1: 1.03$, indicating that GGS-NPs are on the same order of magnitude brightness as silica-gold nanoshells, which have already demonstrated success as in vivo multiphoton contrast agents. ${ }^{21}$ When excitation power was increased to $10 \mathrm{~mW}$, the brightness ratio of GGSNPs to nanoshells increased to 2.57:1. The damage threshold for silica-gold nanoshells has been reported as $4.5 \mathrm{~mW}$ and therefore, it is likely that the reduction in silica-gold nanoshell luminescence was a result of particle melting. ${ }^{21}$ The GGS-NPs appear to have a higher damage threshold than silica-gold nanoshells, indicating they may convert more incident multiphoton energy to luminescence than to heat making them more suitable for combined diagnostic and therapeutic agents.

\section{Antibody-functionalized GGS-NPs enable imaging and thermal ablation of targeted breast cancer cells}

The application of GGS-NPs as dual agents for cancer imaging and treatment was investigated by utilizing low laser powers $(1 \mathrm{~mW})$ to image cancerous cells and high laser powers $(50 \mathrm{~mW})$ to induce cell death. To facilitate binding with SK-BR-3 breast carcinoma cells, GGS-NPs were functionalized with anti-HER2 antibodies (NeoMarkers) and backfilled with mPEG-SH (Laysan Bio, Inc.) as a blocking agent to prevent non-specific binding. Control GGS-NPs were coated with mPEG-SH either with or without anti-IgG antibodies. Results of two-photon microscopy performed with $1 \mathrm{~mW}$ incident power are displayed in Figure 2, where Figure 2a displays the TPL signal (observed only for anti-HER2 functionalized nanoparticles attached to SK-BR-3 cells), Figure $2 b$ is a brightfield (BF) image of the same field of view, and Figure $2 \mathrm{c}$ is an overlay of the TPL and BF images. Successful targeting of SK-BR-3 cells with anti-HER2 functionalized GGS-NPs was demonstrated by the increased TPL signal intensity versus the controls as shown in Figure 2a. At this laser power, SK-BR-3 cells alone (samples incubated with PBS) did not exhibit a luminescent signal, thus any luminescence observed can be attributed to the presence of GGS-NPs. Cells that were incubated with nanoparticles coated with nonspecific antibodies or with only mPEG-SH could not be discerned thereby verifying the specific targeting of anti-HER2 functionalized nanoparticles to SK-BR-3 cells.

For nanoparticle assisted-laser therapy the samples were repositioned and laser power was adjusted from $1 \mathrm{~mW}$ to $50 \mathrm{~mW}$. Figure 3 displays the results of the viability/ cytotoxicity assay performed after exposing cells to the 


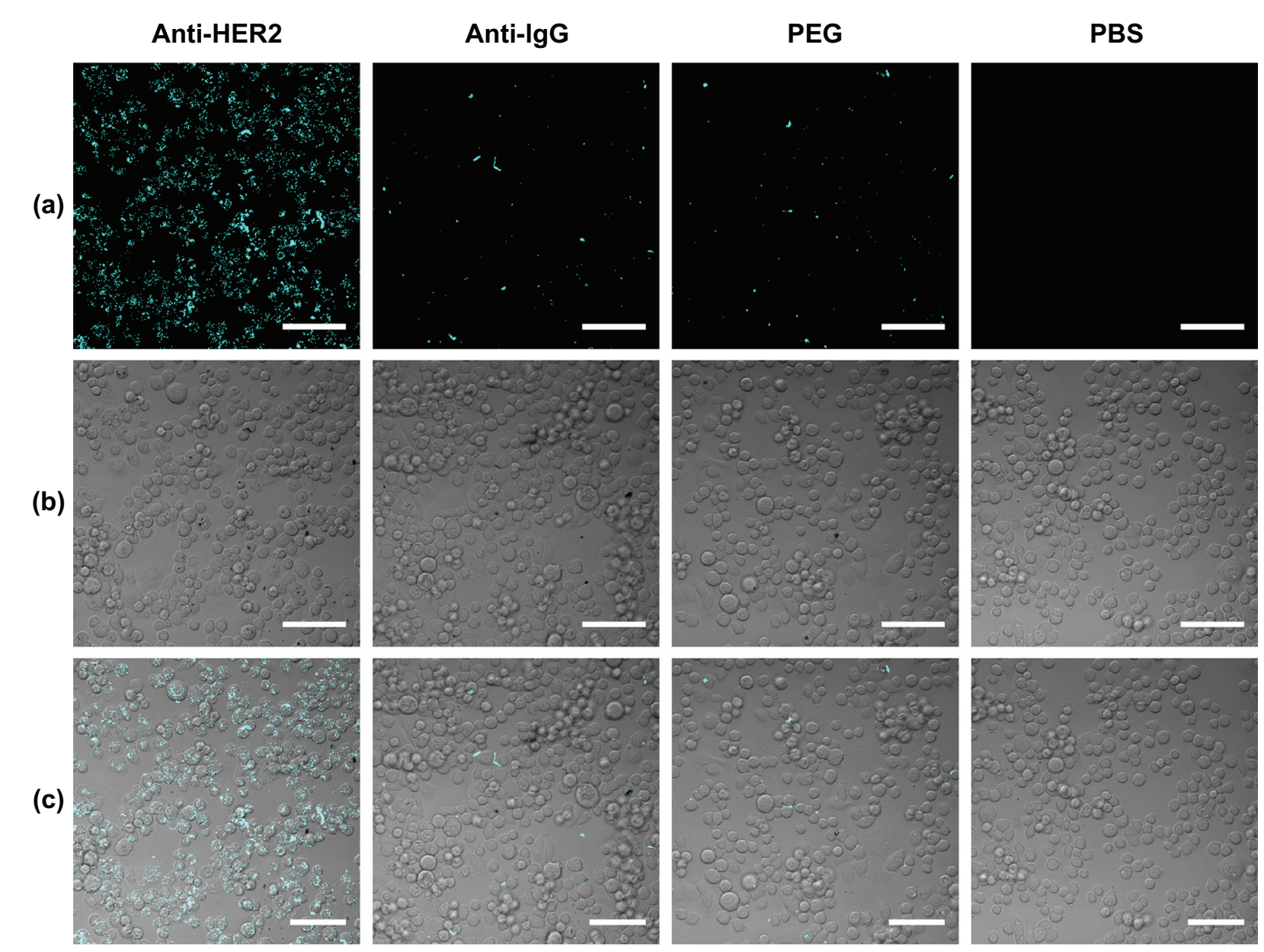

Figure 2 a) Two-photon induced photoluminescence images of SK-BR-3 cells exposed to I $\mathrm{mW}$ with the pulsed laser tuned to $800 \mathrm{~nm}$. b) Brightfield images of SK-BR-3 cells in the same field-of-view as the luminescence images. c) Overlay of images (a) and (b), showing that luminescence was confined to cells targeted with anti-HER2 goldgold sulfide nanoparticles. Scale bar $=100 \mu \mathrm{m}$.

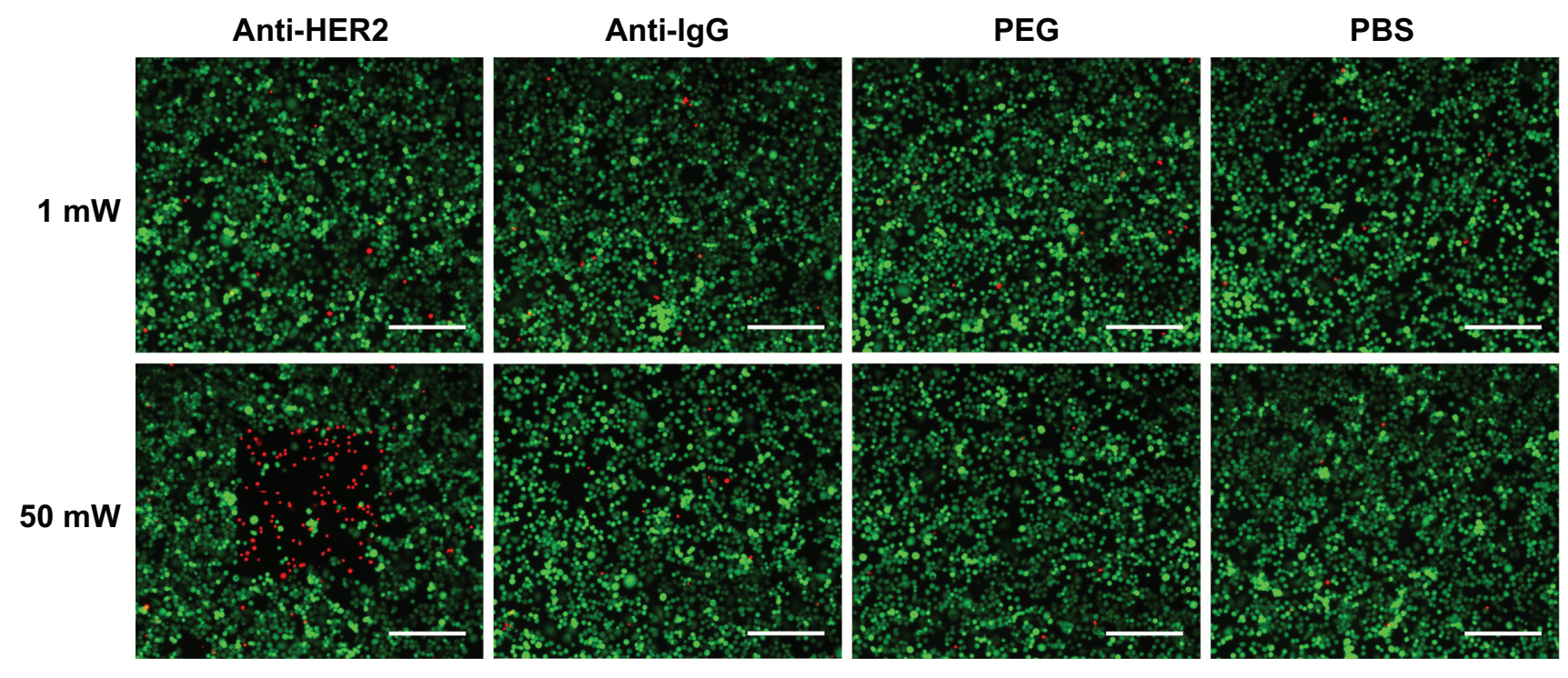

Figure 3 Calcein AM staining indicated that cancerous cells remained viable (evidenced by green fluorescent signal) when exposed to I mW laser power, regardless of nanoparticle presence. At $50 \mathrm{~mW}$ laser output a red fluorescent ethidium homodimer-I signal indicative of membrane damage was observed in cells exposed to anti-HER2 functionalized GGS-NPs only where the laser was applied. Laser exposure alone was harmless to cells, as was laser exposure combined with nonspecifically targeted nanoparticles. Scale bar $=250 \mu \mathrm{m}$. 


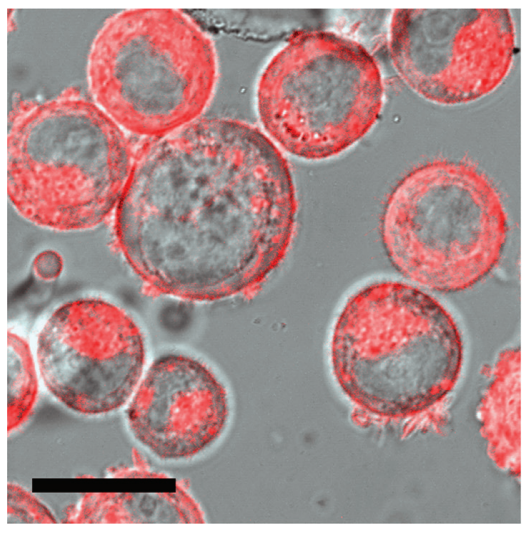

$\mathrm{T}=\mathbf{0} \mathrm{sec}$

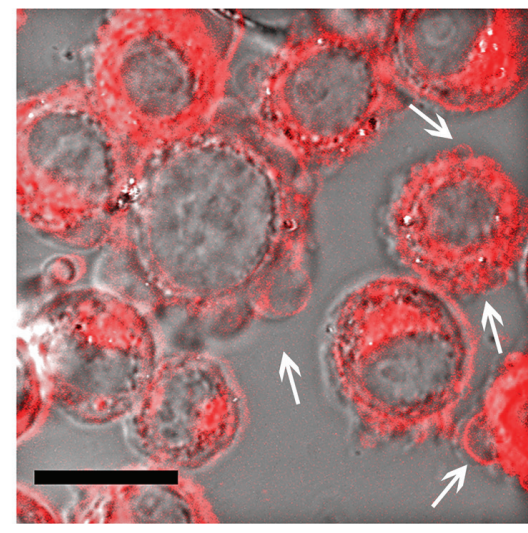

$\mathrm{T}=10 \mathrm{sec}$

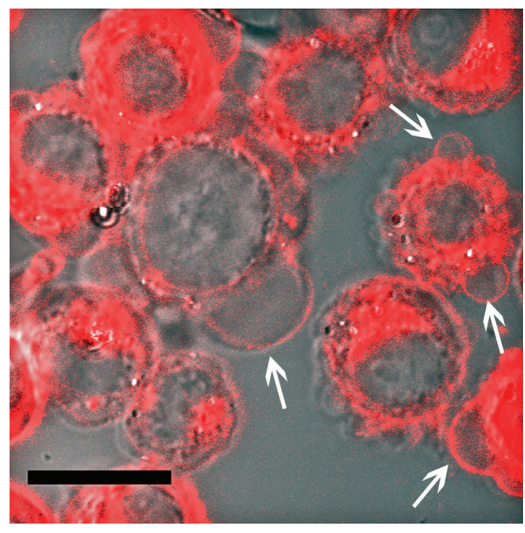

$\mathbf{T}=30 \mathrm{sec}$

Figure 4 Time-lapse photography of SK-BR-3 cells exposed to anti-HER2 functionalized GGS-NPs and 50 mW laser power. The fluorescent red Dil membrane stain indicates regions of membrane blebbing generated by localized hyperthermia, with examples depicted by white arrows. Scale bar $=20 \mu \mathrm{m}$.

nanoparticles and laser. No loss in cell viability was observed in samples exposed to $1 \mathrm{~mW}$ laser power indicating that $1 \mathrm{~mW}$ $\left(0.96 \mathrm{~J} / \mathrm{cm}^{2}\right)$ is not enough power to induce localized heating of the nanoparticles in this time frame and can be safely implemented to image nanoparticle-targeted cells. Using 50 $\mathrm{mW}$ laser power $\left(48.1 \mathrm{~J} / \mathrm{cm}^{2}\right)$ samples exposed to irradiation only or to irradiation and control nanoparticles remained viable, while cell death was induced by thermal damage when cells were exposed to irradiation and anti-HER 2 coated GGSNPs, as indicated by red EthD-1 fluorescence in the squareshaped region where the laser beam was raster-scanned across the sample (Figure 3). Cells outside the laser path remained viable, demonstrating that the anti-HER2 coated GGS-NPs alone were not toxic. This is in keeping with results of prior studies that have examined and established the compatibility of GGS-NPs. ${ }^{31,32}$

\section{Thermal ablation compromises membrane integrity to induce cell death}

The results of the Calcein AM/Ethidium homodimer-1 live/dead stain following laser exposure suggested that one mechanism of cell death was loss of membrane integrity caused by localized heating of the nanoparticles. Ethidium homodimer-1 is excluded from cells with an intact membrane; thus, the fluorescent EthD-1 signal observed in targeted SK-BR-3 cells exposed to the $50 \mathrm{~mW}$ laser indicated that membrane integrity was compromised during photothermal therapy. To observe changes in membrane structure, SK-BR-3 cells were labeled with $5 \mu \mathrm{M}$ DiI (Molecular Probes) for 10 minutes at $37^{\circ} \mathrm{C}$ prior to incubation with the anti-HER 2 nanoparticles and subsequent laser treatment as described before. Targeted, DiI-labeled cells were exposed to high laser power $(50 \mathrm{~mW})$ and imaged with high-resolution $(63 \mathrm{X}, \mathrm{NA}=1.4)$ time-lapse photography to monitor changes in membrane morphology. For this laser power, pixel dwell time, and numerical aperture the fluence was calculated to be $\sim 30 \mathrm{~J} / \mathrm{cm}^{2}$. A $543 \mathrm{~nm}$ laser was used for DiI excitation while GGS-NPs were simultaneously excited with the pulsed $810 \mathrm{~nm}$ Ti:Sapphire laser. Membrane morphology appeared normal before the laser was applied ( $\mathrm{t}=0$ seconds), initial signs of membrane blebbing appeared within 10 seconds, and extensive damage due to hyperthermia occurred within 30 seconds (Figure 4). Control cells exposed to only the $50 \mathrm{~mW}$ laser did not display signs of membrane injury. During this study it was noted that constant excitation with the $50 \mathrm{~mW}$ pulsed laser caused the TPL signal observed from the GGS-NPs to diminish over time, which is consistent with the results of Huff et al who reported decreased signals for nanorods after prolonged exposure to a continuous wave laser operating between 7.5 and $60 \mathrm{~mW} \cdot{ }^{10}$ This loss in signal is likely due to restructuring/ melting of the nanoparticles, although further studies need to be performed to confirm this hypothesis.

\section{Discussion}

Multifunctional nanoparticles have potential to fulfill the need for novel methods to detect and treat neoplasia that thoroughly eliminate disease and improve survival while also minimizing side effects. The objective of this study was to demonstrate that GGS-NPs can be used simultaneously as contrast and therapeutic agents using conventional multiphoton microscopy. In the foreseeable future, this technology will be limited to applications where the tumor is easily acces- 
sible due to the restricted penetration depth of pulsed laser light and therefore we have used breast carcinoma as a model system. Epidermal growth factor receptor 2 (HER2) is amplified in approximately $30 \%$ of breast cancer cases and is associated with a poor prognosis clinically, ${ }^{40}$ so it is an excellent target for novel anti-cancer agents. We showed that GGS-NPs can be specifically targeted towards SK-BR-3 breast carcinoma cells in vitro by functionalizing the gold surface with anti-HER2 antibodies, and that these targeted cells can be visualized using the two-photon induced luminescence of GGS-NPs under excitation with a low intensity pulsed NIR laser. Using a higher intensity of the laser induced nanoparticle heating leading to photothermal ablation of targeted cancer cells caused by membrane blebbing. This study provided a proof-of-concept that GGS-NPs used in conjunction with multiphoton microscopy can provide the ability to "see-and-treat" tumors in a single setting.

GGS-NPs offer several advantages that render them attractive among the growing list of gold-based nanoparticle regimens for cancer management. As previously mentioned, GGS-NPs are smaller than silica-gold nanoshells and nanorods, two of the most thoroughly studied gold-based nanotherapeutics, which should correlate with improved stability and enhanced tumor delivery in vivo. ${ }^{33,41}$ They also do not require capping with surfactants during synthesis which is beneficial not only because it eliminates toxicity concerns such as those associated with the cetyltrimethylammonium bromide (CTAB) surfactant used to prepare nanorods, ${ }^{42}$ but also because it leaves the gold surface available for conjugation to biomolecules. In this work we provided the first demonstration of tethering antibodies to GGS-NPs by utilizing the available gold. Another advantage of GGS-NPs is that they absorb light more efficiently than nanoshells which should yield enhanced imaging and therapy. Though GGS-NPs have been studied for many years, we have only recently described their application in photothermal cancer therapy. ${ }^{33}$ In that work, a continuous wave laser was used to excite the GGS-NPs while in this work a pulsed laser was employed. Use of a pulsed laser not only provides a new imaging capability by producing TPL from the nanoparticles; it also enables simultaneous therapy and this is the first paper to report combined imaging and photothermal therapy with these nanoparticles. It should be noted that use of a high intensity pulsed laser also allows therapy to be achieved more rapidly and with lower total energy dosages than required for continuous lasers, which should cause significantly less heating and damage to surrounding tissue in vivo.
The main advantage of two-photon microscopy in medical imaging is the ability to provide sub-cellular resolution at depths of up to several hundred microns in tissue. ${ }^{17}$ Advancement of this technology to the clinical setting is currently hindered by the cost and size of commercially available multiphoton microscopes; however, the price of femtosecond pulsed lasers should decrease as they become more commonly used and research to miniaturize two-photon microscopes and reduce their complexity is ongoing. Recent successes in development of two-photon endoscopes were achieved by incorporating microelectromechanical systems (MEMS) scanning mirrors into the microscope design. ${ }^{43,44}$ Specifically, Piyawattanametha et al have fabricated a lightweight (2.9 g), MEMS-based two-photon microscope and demonstrated its ability to track individual red blood cells flowing in neocortical microvasculature of adult mice, establishing the future potential for two-photon imaging in vivo. ${ }^{45}$ With further development, multiphoton microscopy combined with dual imaging and therapy GGS-NPs could provide an effective method to pinpoint and treat specific sites following initial tumor detection with wide-field imaging modalities. In one potential application, TPL of targeted nanoparticles could be used to indicate tumor margin status and any suspicious cells located could be eliminated by employing higher intensity photoablation, removing the need for biopsies and additional surgery. This ability to "see-and-treat" would be particularly beneficial when preservation of normal tissue surrounding neoplastic regions is critical.

\section{Conclusion}

The data presented confirms that NIR-absorbing GGS-NPs have properties that render them suitable as a multifunctional agent for cancer management using multiphoton microscopy. Nanoparticles functionalized with anti-HER2 antibodies bound effectively to SK-BR-3 breast carcinoma cells, while those coated with anti-IgG or mPEG-SH did not attach to cancer cells. Luminescence emitted by GGS-NPs upon excitation with a pulsed laser resulted from a two-photon absorption process and was as bright as luminescence emitted from silica-gold nanoshells. At $1 \mathrm{~mW}$ laser power, SK-BR-3 cells labeled with anti-HER2 GGS-NPs were safely visualized and upon increasing laser power to $50 \mathrm{~mW}$ cell death was induced following membrane blebbing. Nanoparticle heating and subsequent cell death was confined to the area where the laser beam was raster-scanned across the sample. Irradiation alone at either power did not generate changes in cell morphology or cause loss of viability. 
Our results indicate that GGS-NPs are an appropriate choice of combined therapeutic and diagnostic (theranostic) agent for use with multiphoton microscopy. GGS-NPs decorated with antibodies can provide enhanced contrast of targeted cancer cells versus non-targeted healthy cells at low incident powers; if imaging results suggest a region is highly malignant, a higher power laser can then be applied to induce localized heating of particles and subsequent hyperthermic damage to the suspicious lesion. Having the ability to visualize and treat tumor cells with high precision will improve cancer management by minimizing damage to normal tissue surrounding neoplastic regions. In addition, removing the waiting period between time of detection and time of treatment will prevent increases in tumor burden that cause eradication of the disease to become more difficult.

\section{Acknowledgments/disclosures}

This work was funded by the National Science Foundation Center for Biological and Environmental Nanotechnology (EEC-0118007 and EEC-0647452). ES Day is supported by a National Science Foundation Graduate Research Fellowship, LR Bickford is supported by a National Defense Science and Engineering Graduate Fellowship, and JH Slater is supported by a KCGCC-NIH Nanobiology Training Fellowship. The authors thank Ying Hu of the Department of Bioengineering at Rice University for assistance with TEM and Andrew Coughlin of the Department of Bioengineering at Rice University for assistance with TEM and dynamic light scattering experiments. JL West has a financial interest in Nanospectra Biosciences, Inc.

\section{References}

1. Jemal A, Siegel R, Ward E, et al. Cancer statistics, 2006. CA Cancer J Clin. 2006;56(2):106-130.

2. Ferrari M. Cancer nanotechnology: opportunities and challenges. Nat Rev Cancer. 2005;5(3):161-171.

3. Weissleder R. A clearer vision for in vivo imaging. Nat Biotechnol. 2001;19(4):316-317.

4. Gobin AM, Lee MH, Halas NJ, James WD, Drezek RA, West JL. Near-infrared resonant nanoshells for combined optical imaging and photothermal cancer therapy. Nano Lett. 2007;7(7):1929-1934.

5. Hirsch LR, Stafford RJ, Bankson JA, et al. Nanoshell-mediated nearinfrared thermal therapy of tumors under magnetic resonance guidance. Proc Natl Acad Sci U S A. 2003;100(23):13549-13554.

6. Loo C, Lowery A, Halas N, West J, Drezek R. Immunotargeted nanoshells for integrated cancer imaging and therapy. Nano Lett. 2005;5(4):709-711.

7. Lowery AR, Gobin AM, Day ES, Halas NJ, West JL. Immunonanoshells for targeted photothermal ablation of tumor cells. Int J Nanomedicine. 2006;1(2):149-154.

8. O’Neal DP, Hirsch LR, Halas NJ, Payne JD, West JL. Photo-thermal tumor ablation in mice using near infrared-absorbing nanoparticles. Cancer Lett. 2004;209(2):171-176.
9. Huang X, El-Sayed IH, Qian W, El-Sayed MA. Cancer cell imaging and photothermal therapy in the near-infrared region by using gold nanorods. J Am Chem Soc. 2006;128(6):2115-2120.

10. Huff TB, Tong L, Zhao Y, Hansen MN, Cheng JX, Wei A. Hyperthermic effects of gold nanorods on tumor cells. Nanomedicine (Lond). 2007;2(1):125-132.

11. Takahashi H, Niidome T, Nariai A, Niidome Y, Yamada S. Gold nanorodsensitized cell death: microscopic observation of single living cells irradiated by pulsed near-infrared laser light in the presence of gold nanorods. Chem Lett. 2006;35(5):500-501.

12. Tong L, Zhao Y, Huff TB, Hansen MN, Wei A, Cheng JX. Gold nanorods mediate tumor cell death by compromising membrane integrity. Adv Mater. 2007;19(20):3136-3141.

13. Chen J, Wang D, Xi J, et al. Immuno gold nanocages with tailored optical properties for targeted photothermal destruction of cancer cells. Nano Lett. 2007;7(5):1318-1322.

14. Wang YW, Xie XY, Wang XD, et al. Photoacoustic tomography of a nanoshell contrast agent in the in vivo rat brain. Nano Lett. 2004; 4(9):1689-1692.

15. Song KH, Kim CH, Cobley CM, Xia Y, Wang LV. Near-infrared gold nanocages as a new class of tracers for photoacoustic sentinel lymph node mapping on a rat model. Nano Lett. 2009;9(1):183-188.

16. Lu W, Huang Q, Ku G, et al. Photoacoustic imaging of living mouse brain vasculature using hollow gold nanospheres. Biomaterials. 2010;31(9):2617-2626.

17. Zipfel WR, Williams RM, Webb WW. Nonlinear magic: multiphoton microscopy in the biosciences. Nat Biotechnol. 2003;21(11): 1369-1377.

18. Boyd GT, Yu ZH, Shen YR. Photoinduced luminescence from the noble metals and its enhancement on roughened surfaces. Phys Rev B. 1986;33(12):7923-7936.

19. Imura K, Nagahara T, Okamoto H. Near-field two-photon-induced photoluminescence from single gold nanorods and imaging of plasmon modes. J Phys Chem B. 2005;109(27):13214-13220.

20. Bickford L, Sun J, Fu K, et al. Enhanced multi-spectral imaging of live breast cancer cells using immunotargeted gold nanoshells and two-photon excitation microscopy. Nanotechnology. 2008; 19(31):315102-315200.

21. Park J, Estrada A, Sharp K, et al. Two-photon-induced photoluminescence imaging of tumors using near-infrared excited gold nanoshells. Opt Express. 2008;16(3):1590-1599.

22. Wang H, Huff TB, Zweifel DA, et al. In vitro and in vivo two-photon luminescence imaging of single gold nanorods. Proc Natl Acad Sci US A. 2005;102(44):15752-15756.

23. Durr NJ, Larson T, Smith DK, Korgel BA, Sokolov K, Ben-Yakar A. Two-photon luminescence imaging of cancer cells using molecularly targeted gold nanorods. Nano Lett. 2007;7(4):941-945.

24. Black KC, Kirkpatrick ND, Troutman TS, et al. Gold nanorods targeted to delta opioid receptor: plasmon-resonant contrast and photothermal agents. Mol Imaging. 2008;7(1):50-57.

25. Tong L, He W, Zhang YS, Zheng W, Cheng JX. Visualizing systemic clearance and cellular level biodistribution of gold nanorods by intrinsic two-photon luminescence. Langmuir. 2009;25(21): 12454-12459.

26. Zhou HS, Honma I, Komiyama H, Haus JW. Controlled synthesis and quantum-size effect in gold-coated nanoparticles. Phys Rev B. 1994;50(16):12052-12056.

27. Norman TJ, Grant CD, Magana D, et al. Near infrared optical absorption of gold nanoparticle aggregates. J Phys Chem B. 2002;106(28): 7005-7012.

28. Zhang JZ, Schwartzberg AM, Norman T, et al. Comment on "Gold nanoshells improve single nanoparticle molecular sensors". Nano Lett. 2005;5(4):809-810.

29. Raschke G, Brogl S, Susha AS, et al. Reply to "Comment on 'Gold nanoshells improve single nanoparticle molecular sensors" ". Nano Lett. 2005;5(4):811-812. 
30. Schwartzberg AM, Grant CD, van Buuren T, Zhang JZ. Reduction of $\mathrm{HAuCl}_{4}$ by $\mathrm{Na}_{2} \mathrm{~S}$ revisited: the case for Au nanoparticle aggregates and against $\mathrm{Au}_{2} \mathrm{~S} / \mathrm{Au}$ core/shell particles. J Phys Chem C. 2007;111(25):8892-8901.

31. Huang XL, Zhang B, Ren L, et al. In vivo toxic studies and biodistribution of near infrared sensitive $\mathrm{Au}-\mathrm{Au}_{2} \mathrm{~S}$ nanoparticles as potential drug delivery carriers. J Mater Sci Mater Med. 2008;19(7):2581-2588.

32. Ren L, Huang XL, Zhang B, et al. Cisplatin-loaded $\mathrm{Au}-\mathrm{Au}_{2} \mathrm{~S}$ nanoparticles for potential cancer therapy: cytotoxicity, in vitro carcinogenicity, and cellular uptake. J Biomed Mater Res A. 2008;85(3): 787-796.

33. Gobin AM, Watkins EM, Quevedo E, Colvin VL, West JL. Nearinfrared-resonant gold/gold sulfide nanoparticles as a photothermal cancer therapeutic agent. Small. 2010;6(6):745-752.

34. Kraus MH, Popescu NC, Amsbaugh SC, King CR. Overexpression of the EGF receptor-related proto-oncogene erbB-2 in human mammary tumor cell lines by different molecular mechanisms. EMBO J. 1987; 6(3):605-610.

35. Averitt RD, Westcott SL, Halas NJ. Linear optical properties of gold nanoshells. J Opt Soc Am B. 1999;16(10):1824-1832.

36. Oldenburg SJ, Averitt RD, Westcott SL, Halas NJ. Nanoengineering of optical resonances. Chem Phys Lett. 1998;288(2-4):243-247.

37. Sau TK, Murphy CJ. Seeded high yield synthesis of short Au nanorods in aqueous solution. Langmuir. 2004;20(15):6414-6420.
38. Zweifel DA, Wei A. Sulfide-arrested growth of gold nanorods. Chem Mater. 2005;17(16):4256-4261.

39. Chen J, Wiley B, Li ZY, et al. Gold nanocages: engineering their structure for biomedical applications. Adv Mater. 2005;17:2255-2261.

40. Slamon DJ, Clark GM, Wong SG, Levin WJ, Ullrich A, McGuire WL. Human breast cancer: correlation of relapse and survival with amplification of the HER-2/neu oncogene. Science. 1987;235(4785):177-182.

41. Zhang GD, Yang Z, Lu W, et al. Influence of anchoring ligands and particle size on the colloidal stability and in vivo biodistribution of polyethylene glycol-coated gold nanoparticles in tumor-xenografted mice. Biomaterials. 2009;30(10):1928-1936.

42. Niidome T, Yamagata M, Okamoto Y, et al. PEG-modified gold nanorods with a stealth character for in vivo applications. $J$ Control Release. 2006;114(3):343-347.

43. Jung WY, Tang S, McCormic DT, et al. Miniaturized probe based on a microelectromechanical system mirror for multiphoton microscopy. Opt Lett. 2008;33(12):1324-1326.

44. Hoy CL, Durr NJ, Chen P, et al. Miniaturized probe for femtosecond laser microsurgery and two-photon imaging. Opt Express. 2008; 16(13):9996-10005.

45. Piyawattanametha $\mathrm{W}$, Cocker ED, Burns LD, et al. In vivo brain imaging using a portable $2.9 \mathrm{~g}$ two-photon microscope based on a microelectromechanical systems scanning mirror. Opt Lett. 2009; 34(15):2309-2311.
International Journal of Nanomedicine

\section{Publish your work in this journal}

The International Journal of Nanomedicine is an international, peerreviewed journal focusing on the application of nanotechnology in diagnostics, therapeutics, and drug delivery systems throughout the biomedical field. This journal is indexed on PubMed Central, MedLine, CAS, SciSearch ${ }^{\circledR}$, Current Contents ${ }^{\circledR} /$ Clinical Medicine,

\section{Dovepress}

Journal Citation Reports/Science Edition, EMBase, Scopus and the Elsevier Bibliographic databases. The manuscript management system is completely online and includes a very quick and fair peer-review system, which is all easy to use. Visit http://www.dovepress.com/ testimonials.php to read real quotes from published authors. 